\title{
EDUCACIÓN PARA LA MUERTE Y EL MORIR PARA UNA VIDA PLENA: APRENDER A MORIR PARA APRENDER A VIVIR
}

\author{
Marta Domínguez Martínez \\ Educadora Social \\ martadomin8@gmail.com \\ Ana Isabel Isidro de Pedro \\ Universidad de Salamanca \\ anyis@usal.es
}

Recepción Artículo: 13 marzo 2020 Admisión Evaluación: 17 marzo 2020

Informe Evaluador 1: 1 abril 2020

Informe Evaluador 2: 7 abril 2020

Aprobación Publicación: 20 abril 2020

\section{RESUMEN}

La muerte es un acontecimiento que nos llega, por orden natural o no, indefectiblemente al final de nuestra vida. Pudiera esto parecer una perogrullada, pero es la única certeza que realmente tenemos. No todas las culturas afrontan, ofician, conmemoran ni representan psicosocialmente la muerte de igual manera y, en consecuencia, no todas las personas pasan por el mismo duelo, ni tienen las mismas actitudes, pensamientos y sentimientos hacia el tema. En nuestra sociedad la muerte es un tema tabú y constituye una de las fuentes de angustia más significativas e importantes. Este trabajo presenta un estudio realizado en una muestra de personas mayores de edad $(n=200)$, distribuida en cinco rangos etarios, para intentar determinar la etapa vital, si es que la hubiere, en la que se pasa de temer a la muerte a aceptarla ¿Aprendemos a morir en los últimos momentos de la vida 0 , tal vez, ni siquiera entonces? En este sentido, se ha utilizado un cuestionario de elaboración propia, cuyos ítems están relacionados con el tipo de pensamientos hacia la muerte, el reparo a hablar de ella, el miedo a la muerte de los seres queridos, a morir solo, a la pérdida, a la muerte repentina, la ansiedad ante el tema, etc. Finalmente, se hace mención a la educación para la muerte como herramienta para asumir y aceptar la misma, con el objetivo de llevar una vida plena y de completo bienestar psicosocial.

Palabras clave: muerte; envejecimiento; aceptación; educación para la muerte

\section{ABSTRACT}

Education for death and dying for a full life: Learning to die to learn to live. Death is an event that comes to us, by natural order or not, unfailingly at the end of our life. This may sound like a truism, but it is the only certainty we really have. Not all cultures confront, officiate, commemorate or psychosocially represent 
death in the same way and, consequently, neither all people go through the same grief, nor do they have the same attitudes, thoughts and feelings towards the matter. In our society, death is a taboo subject and constitutes one of the most significant and important sources of anguish. This work presents a study carried out on a sample of people of legal age $(n=200)$, distributed over five age ranges, to try to determine the life stage, if any, in which one goes from fearing death to accept it. Do we learn to die in the last moments of life or, perhaps, not even then? In this sense, a self-elaborated questionnaire has been used, the items of which are related to the type of thoughts towards death, the qualms about talking about it, the fear to the loved ones death, of dying alone, of loss, to the sudden death, the anxiety about the subject, etc. Finally, a mention is made on the education for death as a tool to assume and accept it, with the aim of leading a full life and full psychosocial well-being.

Keywords: death; aging; acceptance; education for death

\section{INTRODUCCIÓN}

Desde que somos pequeños tenemos un manifiesto contacto con la muerte, además de cercano. En un determinado momento, todos los niños son conscientes de lo que este término abarca por distintas situaciones a las que tienen que hacer frente.

He escogido este tema porque quiero, y realmente necesito, dar un significado a la muerte, a cómo la aceptamos y al porqué se produce dicha aceptación. Considero que es necesario educar para la muerte desde la infancia. Es fundamental aprender a morir para poder vivir plenamente y sin miedo. Debemos ser capaces de hacer de la propia vida algo bello, significativo y profundo.

Podemos determinar que las personas mayores no temen a la muerte, ya que hay una correlación negativa entre edad y ansiedad ante la misma. Es por ello, que en este Trabajo de Fin de Grado queremos verificar que esto ocurre así, que son las personas jóvenes las que más miedo tienen a la muerte o más angustia les crea el hecho de pensar en ella y, por el contrario, las personas mayores tienen aceptada la muerte como tal.

Los más importante de todo quizá se definir el significado de muerte. Según la Real Academia Española (RAE), la muerte es la "cesación o término de la vida"; en el pensamiento tradicional, separación del cuerpo y el alma. En esta primera definición, ya encontramos dos ramas: la forma natural de la muerte y la entendida como forma espiritual; ya que, como bien dicen Martínez, Alonso y Calvo (2001), la muerte se puede considerar como un elemento al que es difícil dar sentido.

Además, e independientemente de cómo pueda entenderse la muerte, podemos hablar de cuatro tipos, los cuales pueden escenificarse en una escalera dado que, para llegar a morir "del todo", tienes que ir pasando por cada una de las muertes mencionadas.

Figura 1. Fases por las que pasa la persona antes de morir

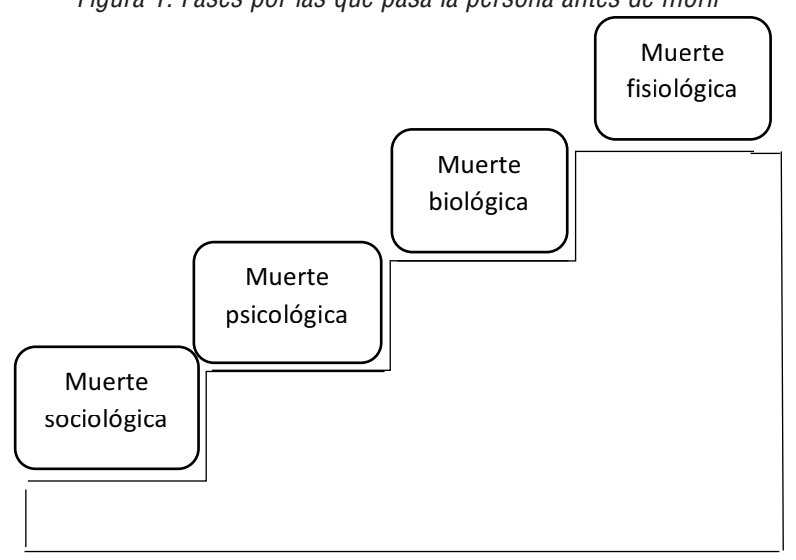


Actualmente, la muerte es considerada un tema tabú en nuestra sociedad. Esto viene dado porque causa, de forma recurrente, ansiedad y miedo, lo cual se hace muy notable en la adolescencia. Por ello, aunque es un tema socialmente rechazado, se pretende que sea personalmente aceptado y, para ello, hay que aprender a morir. Como afirman Serra y Abengózar (1990, p. 147), "el conocimiento de la vida humana lleva implícita la dimensión temporal, y, en ella, el tiempo biográfico nos conduce a la idea del fin, y, por tanto, a la idea de muerte".

Por otro lado, tenemos que hacer referencia a la vejez siendo una etapa de cambios donde se pueden observar las últimas transformaciones de declive que se producen en el ser humano y es, en este momento, cuando comienza a entenderse la muerte o vincularse como algo inevitable, lo cual hace que se produzca una aceptación de la misma.

Todo lo relacionado con la muerte, tiene mucha influencia con la ansiedad y el miedo, o viéndolo de otra manera; la ansiedad y el miedo tienen mucha influencia en la muerte. El temor a la muerte puede llevar a la persona a experimentar un auténtico síndrome de ansiedad ante la misma.

\section{APRENDER A MORIR Y EDUCAR PARA LA MUERTE}

En muchas ocasiones, podemos encontrarnos con personas que se pasan la vida pensando en la muerte en vez de tratar de disfrutar de la misma al máximo. Consideramos que, si desde que somos pequeños, se nos diera la educación correcta para aprender a morir y para hacerle frente de la manera más beneficiosa para nosotros, sabiendo afrontarla sin que eso suponga estados de ansiedad y miedo, la sociedad, en general, sería más feliz de lo que puede serlo a día de hoy.

La mencionada educación para la muerte debería darse desde todos los ámbitos en los que se desarrolla el niño. Hablamos, principalmente, de la escuela y de los hogares familiares. Sería necesaria una educación para que estas figuras (padres y docentes) sepan tratar correctamente el tema del que estamos hablando. Es una evidencia que, en muchas ocasiones, los padres o los familiares más cercanos, no saben cómo tratar estos temas (principalmente por la carga emocional que puede suponer la pérdida de un ser querido), dado que es un tema muy duro y difícil. Por ello, la figura de un maestro, tutor, un educador social u otro profesional especializado, preparado y formado de previamente para ello, es de vital importancia. Es muy necesario alguien que sepa cómo abordar el tema, cómo comunicar una pérdida y cómo acompañar en todo ese proceso, para que no sea considerado como algo a lo que hay que temer, ni realmente negativo, como muchas veces pueden transmitir los familiares al comunicar la noticia.

Es por todo esto, que consideramos que, desde la infancia, es necesaria una introducción plena y profundizada hacia el tema de la muerte. La educación para la muerte seguida de una educación para el morir, son realmente importantes en nuestro día a día. Si sabemos que vamos a morir, lo que conlleva la muerte y los motivos de la misma, vamos a poder disfrutar la vida de una forma más plena, sintiéndonos más completos y con más ganas de vivir, ya que, al final, es el único sentido de la vida.

\section{OBJETIVOS}

El objetivo general que se pretende alcanzar con esta investigación es:

- Identificar a qué edad se pasa de tener miedo hacia la muerte a sentir una aceptación a la misma.

- Por otro lado, y teniendo en cuenta el objetivo general, se pretenden conseguir los siguientes objetivos específicos:

- Determinar en qué rango de edad se tiene más miedo ante la muerte, tanto propia como de los seres queridos.

- Visibilizar cuándo es más probable sentir ansiedad ante temas relacionados con la muerte.

\section{METODOLOGÍA}

Esta investigación pretende determinar la franja etaria, si es que la hubiere, en la que se produce el paso de temer a la muerte a aceptarla. Se utilizará una metodología cuantitativa, mediante la elaboración y aplicación de 
un cuestionario. A través de éste se recogerán todas las respuestas de los ítems planteados para así poder dar respuesta a las cuestiones derivadas de los objetivos.

\section{Muestra}

Se trata de una muestra de personas mayores de edad $(n=200)$, distribuida en cinco rangos etarios (18-35 años; 36-50 años; 51-65 años; 66-75 años; más de 75 años), 40 personas por cada franja de edad.

\section{Instrumento y aplicación}

Para verificar la hipótesis de que son las personas mayores las que menos miedo y ansiedad experimentan hacia la muerte, por la aceptación de la misma, se ha elaborado un cuestionario ad hoc que se encuentra dividido en 4 partes. En la primera parte del cuestionario se requieren datos generales. La segunda parte consta de 5 ítems (escala Tipo Likert) enfocados al propio concepto de la muerte y cómo las personas lo perciben. En el tercer apartado los 9 ítems (escala Tipo Likert) se refieren a la forma de abordar conversaciones sobre la muerte Además, también se quiere comprobar si las personas creen tener control a la hora de hablar sobre esta cuestión. Por otro lado, y para terminar, los 9 ítems (dicotómicos) del último bloque poseen relevancia para el tema, pero no están vinculados con el concepto de muerte propiamente dicho, sino que nos ayudan a determinar la facilidad o dificultad que tienen las personas al lidiar con cuestiones relacionadas con este asunto.

\section{ANÁLISIS DE DATOS Y DISCUSIÓN}

El programa utilizado para el tratamiento y análisis de los 200 cuestionarios ha sido el PSPP.

En primer lugar, es necesario situarnos y comprender qué rango de edad es el que más piensa en la muerte, para después poder continuar profundizando en otros aspectos analizados.

Tabla 1: Media de edad que más piensa en la muerte

\begin{tabular}{||r|r|r|r|r|}
\hline & EDAD & Media & \multicolumn{1}{c|}{$N$} & Desviación Estándar \\
\hline \hline PIENSOMUERTE & $18-35$ & 2.40 & 40.00 & .96 \\
& $36-50$ & 2.45 & 40.00 & .88 \\
& $51-65$ & 2.58 & 40.00 & .75 \\
& $66-75$ & 2.15 & 40.00 & 1.03 \\
& +75 & 1.90 & 40.00 & 1.19 \\
\hline
\end{tabular}

Como se puede observar en la Tabla 1, las personas que más piensan en la muerte son las comprendidas entre 51 y 65 años y las que menos piensan en ella las mayores de 75 años. Además, uno de los saltos más destacados se produce entre el tercer y cuarto rango de edad. En el tercer rango la media es $X=2.58$, mientras que en el cuarto pasa a ser $X=2.15$; la distancia más alejada de su siguiente. Por lo que se podría determinar que en la edad de jubilación (65 años) es cuando se comenzaría a pensar con menos asiduidad en la muerte.

Tabla 2. Media de edad que más miedo tiene a morir

\begin{tabular}{||r|r|r|r|r|}
\hline & EDAD & Media & N & Desviación Estándar \\
\hline \hline MIEDOMORIR & $18-35$ & 2.50 & 40.00 & 1.15 \\
& $36-50$ & 2.75 & 40.00 & 1.06 \\
& $51-65$ & 2.77 & 40.00 & 1.14 \\
& $66-75$ & 2.03 & 40.00 & 1.05 \\
& +75 & 1.95 & 40.00 & 1.22 \\
\hline
\end{tabular}

En la Tabla 2, podemos observar cómo ocurre exactamente lo mismo que en la anterior. Las personas que más miedo tienen a morir son las que se encuentran entre los 51 y los 65 años; y las que menos miedo tienen son las mayores de 75 años. Por otro lado, el salto más grande de una horquilla a otra también es entre la tercera 
y cuarta franja de edad. Las personas entre los 51 y 65 años tienen una media de $X=2.77$ y la siguiente horquilla una media de $X=2.03$, habiendo una distancia realmente alta entre ambas.

Tabla 3. Media de edad que más miedo experimenta al hablar de muerte
\begin{tabular}{|r|r|r|r|r|}
\hline \hline & EDAD & Media & \multicolumn{1}{c|}{$N$} & Desviación Estándar \\
\hline \hline HABLOMIEDO & $18-35$ & 2.17 & 40.00 & 1.15 \\
& $36-50$ & 2.90 & 40.00 & 1.22 \\
& $51-65$ & 2.58 & 40.00 & 1.24 \\
& $66-75$ & 1.88 & 40.00 & 1.14 \\
& +75 & 2.15 & 40.00 & 1.17 \\
\hline
\end{tabular}

En la Tabla 3, como podemos apreciar, las personas que más miedo sienten cuando en una conversación sale el tema de la muerte son las que se encuentran entre los 36 y los 50 años. En este sentido, se podría interpretar como que es la edad en la que comienzan a tener una familia propia, creada por ellos mismos y, por lo tanto, les aterroriza la idea de dejar el mundo y no poder ver crecer a sus hijos, ni poder desarrollar sus objetivos ni planes en la vida.

Tabla 4. Media de edad que más miedo tiene a morir solo

\begin{tabular}{|l|r|r|r|r|}
\hline & EDAD & Media & N & Desviación Estándar \\
\hline \hline MIEDOSOLO & $18-35$ & 2.77 & 40.00 & 1.39 \\
& $36-50$ & 2.90 & 40.00 & 1.39 \\
& $51-65$ & 3.35 & 40.00 & 1.25 \\
& $66-75$ & 2.05 & 40.00 & 1.18 \\
& +75 & 2.38 & 40.00 & 1.33 \\
\hline
\end{tabular}

Hablando ahora del miedo a morir solo (ver Tabla 4), y relacionándolo con la Tabla 2, coincide en que el rango etario que más miedo tiene a morir es, también, el que más miedo tiene a hacerlo solo: de los 51 a los 65 años. Lo cual podría deberse a que se encuentran en la etapa vital coincidente con el "nido vacío", advierten que se van haciendo más mayores, comienzan a tener algunas limitaciones físicas a la hora de realizar determinadas acciones y sienten miedo al abandono, a verse solos.

Tabla 5. Media de edad que más ansiedad experimenta al hablar de la muerte

\begin{tabular}{||r|r|r|r|r|}
\hline & EDAD & Media & N & Desviación Estándar \\
\hline \hline ANHABLARM & $18-35$ & 1.78 & 40.00 & 1.10 \\
& $36-50$ & 2.73 & 40.00 & 1.32 \\
& $51-65$ & 2.30 & 40.00 & 1.14 \\
& $66-75$ & 2.02 & 40.00 & 1.07 \\
& +75 & 2.00 & 40.00 & 1.13 \\
\hline
\end{tabular}

Cuando en una conversación sale el tema de la muerte, las personas que más ansiedad experimentan ante ello son las que tienen entre 36 y 50 años, en contraposición de las que tienen entre 18 y 35 (ver Tabla 5). Este hallazgo es llamativo, porque pasamos del rango que menos ansiedad experimenta al que más lo hace, siendo la diferencia de casi un punto. Esto podría producirse porque en la segunda etapa de edad ya es cuando comenzamos a ser más conscientes de todas las responsabilidades que conlleva "vivir".

Tabla 6. Media de edad que más control refiere tener ante el tema de la muerte

\begin{tabular}{|l|r|r|r|r|}
\hline & EDAD & Media & $N$ & Desviación Estándar \\
\hline \hline CONTROL & $18-35$ & 3.17 & 40.00 & 1.57 \\
& $36-50$ & 2.58 & 40.00 & 1.03 \\
& $51-65$ & 2.98 & 40.00 & 1.10 \\
& $66-75$ & 3.47 & 40.00 & 1.09 \\
& +75 & 3.10 & 40.00 & 1.41 \\
\hline
\end{tabular}


Como podemos apreciar en la Tabla 6 , las personas que menos control refieren tener son las que se encuentran entre 36 y 50 años, con una puntuación de $X=2.58$, coincidiendo con lo comentado en la Tabla 6, que son las personas que más ansiedad experimentan cuando en alguna conversación surge el tema de la muerte. Por lo tanto, parece que se encuentra cierta correlación entre estos resultados.

Es necesario determinar también que, tanto los más jóvenes como los más ancianos, refieren tener un control bastante similar ante el tema, presentando las puntuaciones $X=3.17$ y $X=3.10$ respectivamente.

Como podemos observar en la Figura 2, las personas de más de 75 años son las que menos miedo tienen a la muerte repentina, ya que solo 16 personas del total (40) han marcado un 1 en esta opción. Además, la barra de esta franja de edad va disminuyendo. Por otro lado, las personas que más miedo parecen experimentar ante la muerte repentina son las que se encuentran entre 36 y 50 años, marcando un total de 13 personas la opción de "Muchísimo".

Por ende, se puede apreciar cómo a partir de los 65 años hay un punto divisorio. Las barras de color morado (más de 75 años) y azul (entre 66 y 75 años) disminuyen considerablemente, en contraposición con las barras de color amarillo (entre 18 y 35 años) y verde (entre 36 y 50 años) que lo que hacen es aumentar. Así, en edades tempranas, hasta los 50 años, se tiene un gran miedo a la muerte repentina, por el hecho de no poder despedirse de los allegados y porque se considera que no es "edad para morir".

Figura 2: Miedo a la muerte repentina

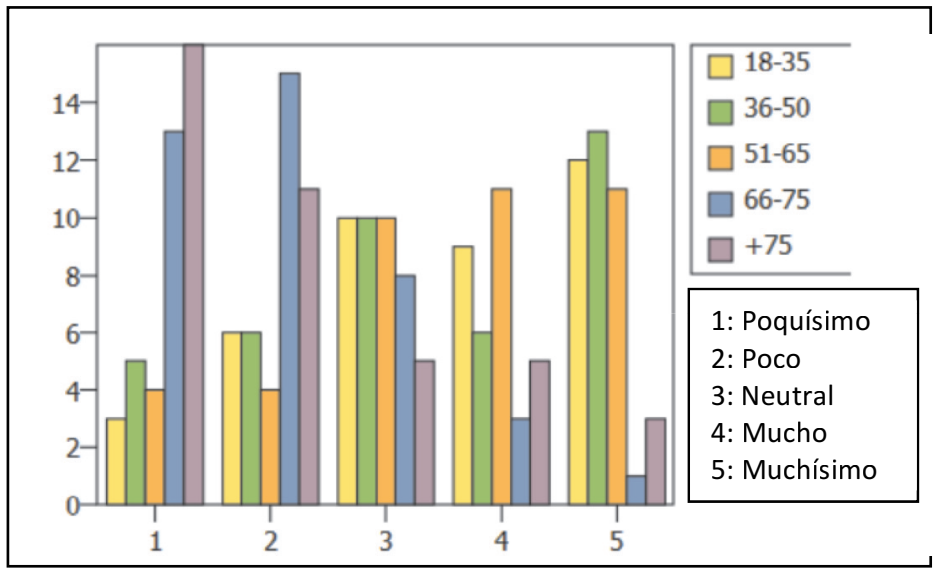

Siguiendo en la misma línea de la Figura anterior, se ha querido determinar también si a las personas les asusta pensar en aquello que dejan al morir (ver Figura 3). 
Figura 3: Miedo al pensar en lo que perderán al morir

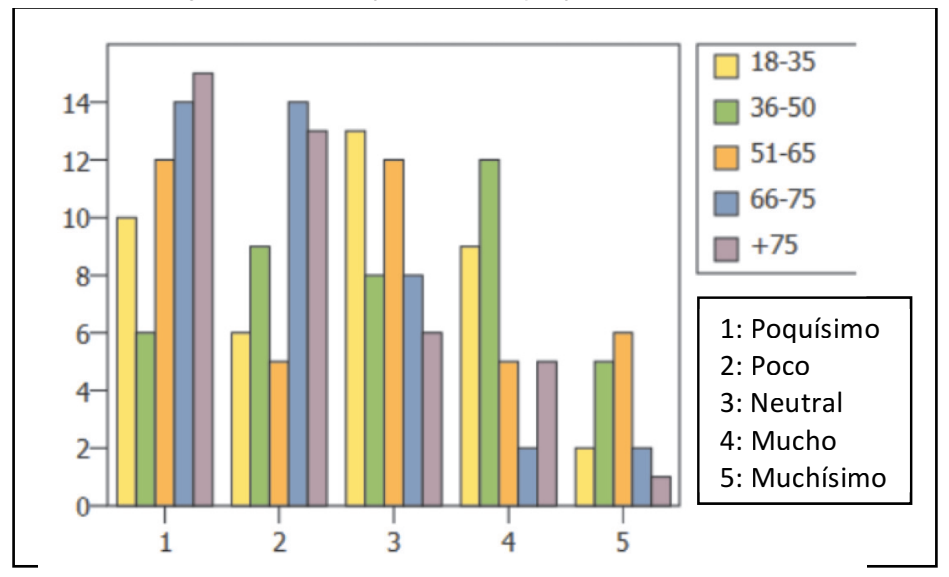

Podemos observar cómo al grupo de personas que menos le asusta lo que puedan perder son las mayores de 75 años; lo cual puede deberse a que ya son conscientes de que, por ley natural, su final está más cerca y tienen que disfrutar de lo que les queda, en vez de lamentarse por lo que ya no podrán hacer. Las que más sobresalen son las comprendidas entre 36 y 50 años, dado que un total de 12 personas han marcado la opción de "Mucho", bajando considerablemente en la siguiente opción (solo 5 personas). De las 200 personas que conforman la muestra, tan solo 16 personas (el 8\% del total) han referido asustarse al pensar en las cosas que pueden perder al morir.

Situándonos en otro aspecto, hay personas que consideran que el tiempo pasa muy rápido, siendo la vida muy corta. Como podemos apreciar en la Figura 4, la mayor parte de la muestra, considera que el tiempo sí pasa muy rápido; constituyendo el 100\% de las personas entre 36 y 50 años los que así lo han hecho saber. Solo encontramos 14 personas (el $7 \%$ del total) que consideran que el ritmo de la vida es normal y no consideran que pase rápido.

Figura 4: El tiempo pasa muy rápido

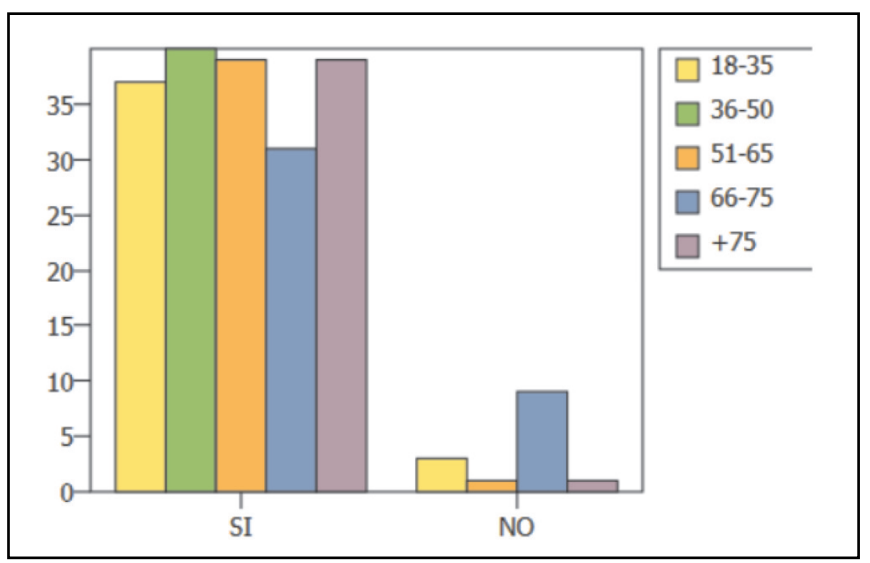


Por otra parte, e íntimamente relacionado con lo antes comentado, quienes han contestado afirmativamente a que consideran que el tiempo pasa rápido han debido contestar que sí tienen miedo de que esto suceda de manera tan veloz. Observando la Figura 5, podemos ver que hay una igualdad entre las personas que tienen miedo de que el tiempo pase rápido y las que no lo tienen; aunque hay más gente en negativo que en positivo (54.5\% frente al $45.5 \%$ respectivamente). Por lo tanto, una gran mayoría piensa que el tiempo pasa realmente rápido (un 93\% de la muestra), pero solo aproximadamente la mitad de la muestra teme a este hecho. Las personas que más miedo refieren tener a esta circunstancia son las que se encuentran entre 51 y 65 años, lo cual puede deberse a que ya han pasado la mitad de la vida y se han dado cuenta fehacientemente de que el tiempo vuela.

Figura 5: Me asusta ver cómo el tiempo pasa tan rápido

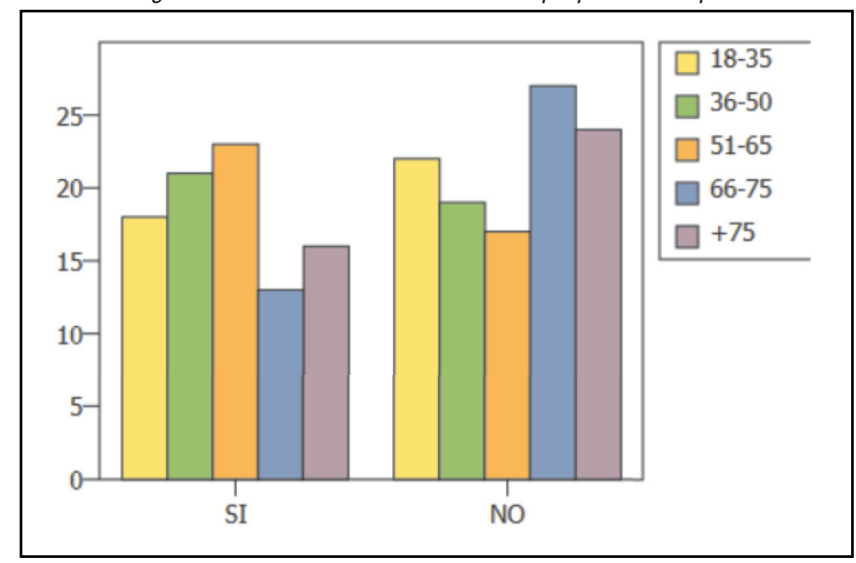

La mayor parte de las personas de la muestra creen que la vida es muy corta, frente a un total de 34 personas (solo el 17\% del total) que no lo creen de esta forma. De estas 34 personas, 16 (el 47\%) tienen entre 18 y 35 años. Se interpreta esta respuesta como una falta de vivencias, es decir, no creen que la vida es muy corta porque todavía no han vivido mucho y creen que van a vivir mucho más (ver Figura 6).

Figura 6: La vida es muy corta

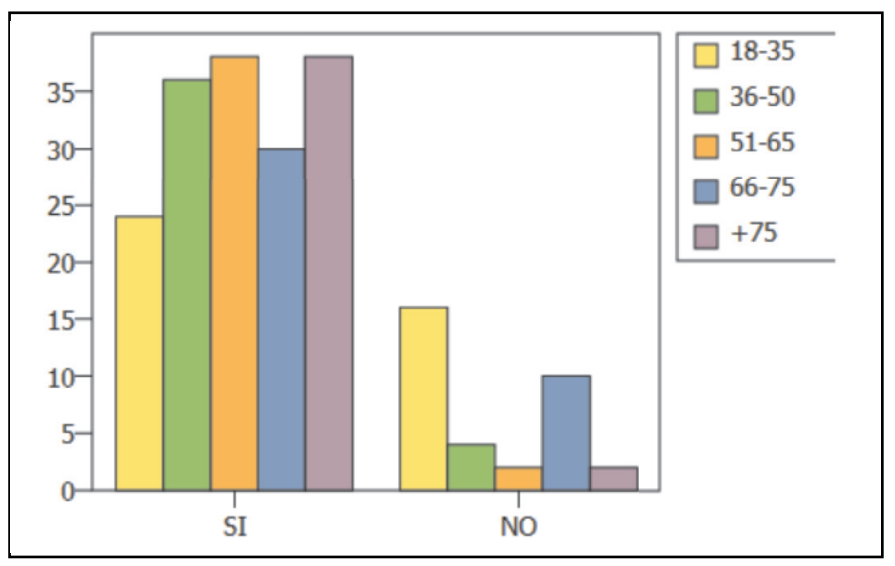


Además de todo lo mencionado, también nos podemos encontrar con personas que ponen imaginación y recrean el tema, anticipando cómo será su muerte. No son muchas, pero las hay (ver Figura 7).

Figura 7: Número de personas que imaginan cómo será su muerte

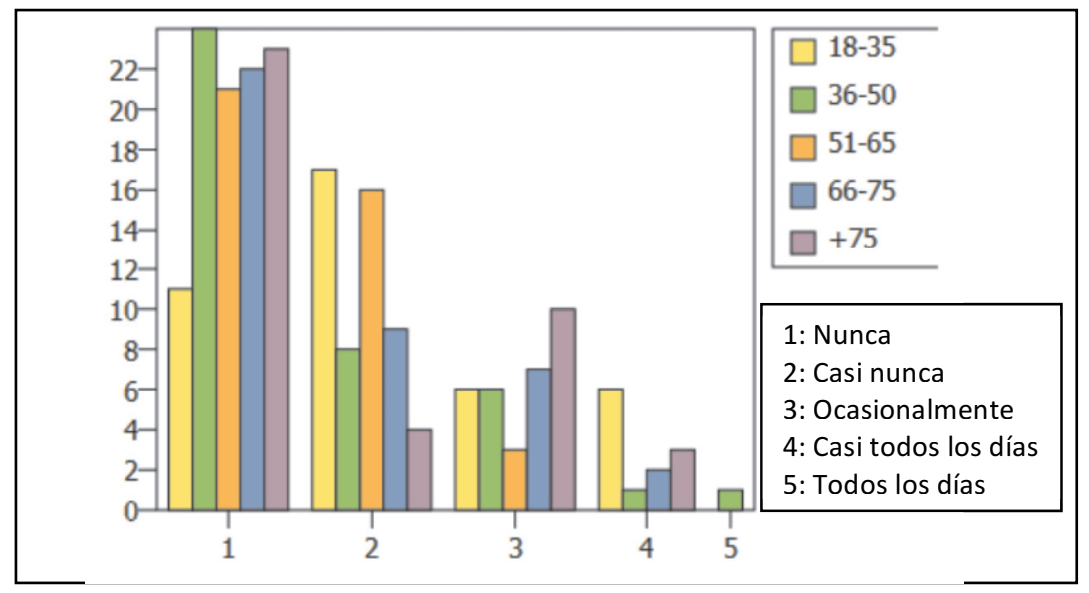

Se observa que la mayor parte de la muestra no imagina nunca cómo será su muerte, siendo solo dos personas de entre 36 y 50 años las que todos los días se imaginan su final. Otro dato curioso que debemos resaltar es que entre las personas que "Nunca" piensan en su muerte, los más jóvenes son los que menos han elegido esta opción, siendo un total de 6 personas de 18 y 35 años los que "Casi todos los días" imaginan cómo será su muerte.

En relación con esta cuestión también se ha querido hacer mención a la muerte de los seres queridos. Con ello, vamos a determinar en qué horquilla de edad se piensa más en la muerte de los más cercanos. Es necesario ver cómo al pensar en otras personas, la cosa cambia notablemente (ver Figura 8). El número de veces que se piensa en la muerte de los demás es mayor al pensamiento en la propia muerte, lo que implica que nos asusta más perder a los demás que dejar de existir en este mundo. Y, como también se advierte, las personas que más piensan en la muerte de los demás (marcando "Casi todos los días" y "Todos los días) son las mayores de 75 años; lo cual, puede deberse a que sus personas amadas ya están mayores y temen poder perderlas, sabiendo que, tarde o temprano, tendrá que llegar ese momento. Porque, como bien dice el exitoso novelista Nicholas Sparks, nunca se está preparado para perder a alguien que amas. 
Figura 8: Número de personas que piensan en la muerte de sus seres queridos

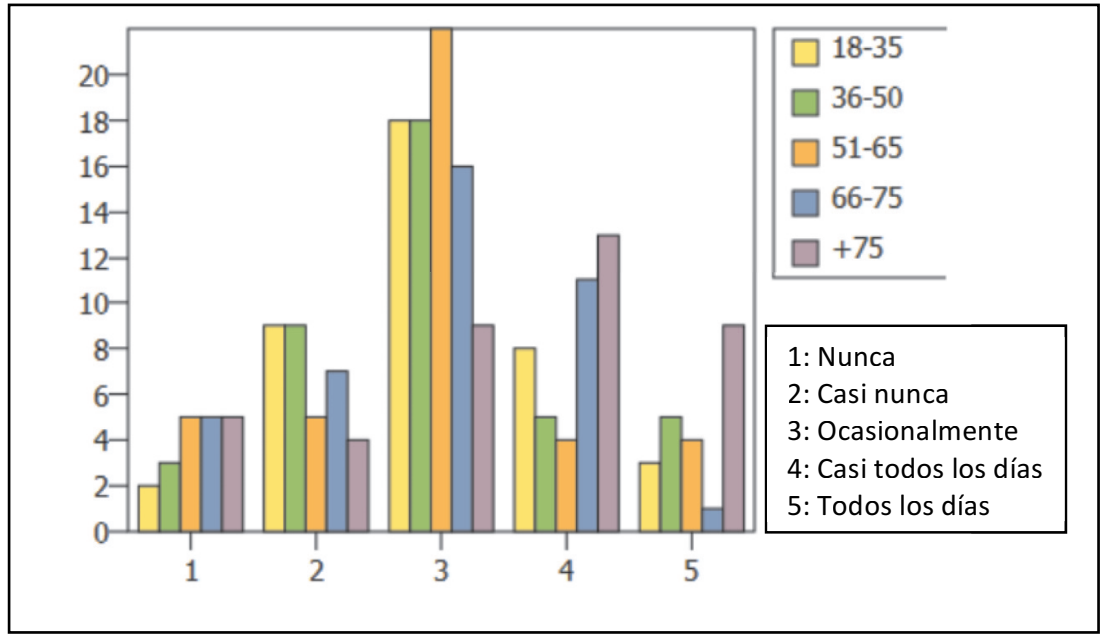

Pero esto no es todo, porque no solo hay personas que piensan en la muerte de los demás, sino que también se asustan ante el hecho de poder perder a alguien; lo cual puede condicionarles mucho en determinados aspectos.

Observando la Figura 9, es evidente que a la mayoría de los sujetos de la muestra les preocupa la pérdida de sus seres queridos. Son pocos los que "Nunca" o "Casi nunca" se asustan por poder perder a alguien, un total de 22 personas, constituyendo el 11\% del total de la muestra. Por lo tanto, encontramos que, en la mayoría de los casos, las personas piensan y se asustan más por la muerte de las personas a las que quieren que por su propia muerte, lo cual podría indicar que estamos menos preparados para perder a alguien que para morir nosotros.

Figura 9: Número de personas que se asustan ante la posibilidad de muerte de sus seres queridos

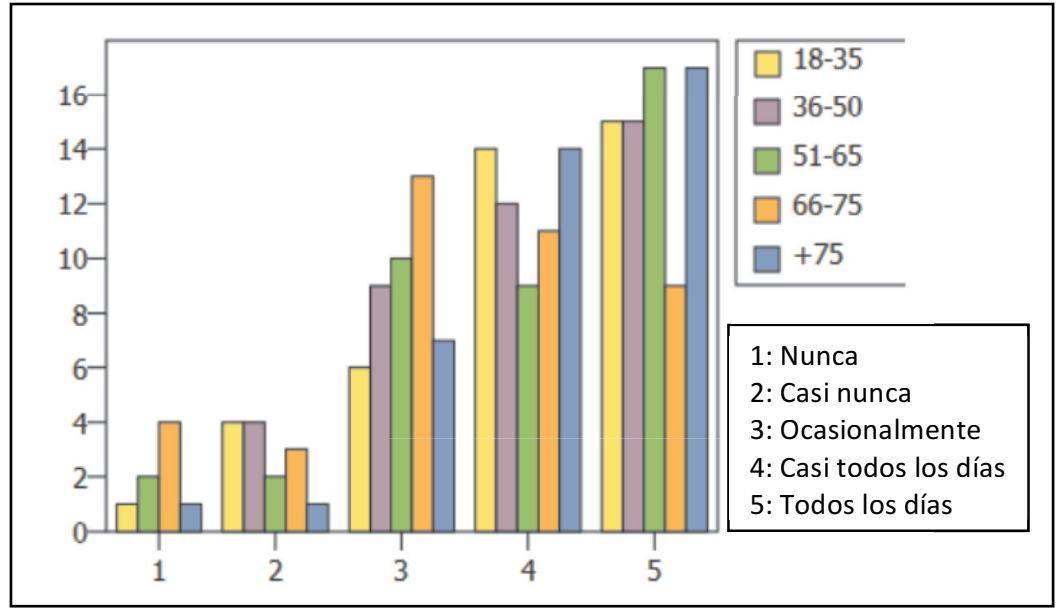


Por último, nos ha parecido interesante estudiar si las personas que conforman la muestra consideran que el resto de personas piensan de la misma forma que ellas en los ítems respondidos (el denominado sesgo de falso consenso o semejanza supuesta). Las conclusiones que se pueden extraer son las siguientes (ver Figura 10).

Es realmente curioso ver cómo un $53,5 \%$ de la muestra cree que todo el mundo tiene la misma visión que él/ella acerca de la muerte, incurriendo en el antedicho sesgo cognitivo. Son las personas comprendidas entre 51 y 65 años quienes más creen que los demás tienen pensamientos similares a los suyos, en contraposición con las que se encuentran entre 66 y 75, que consideran que no todo el mundo tiene la misma visión ante la muerte. Como puede observarse, es este grupo de edad el que más difiere del resto, que consideran, por mayoría, que la gente piensa de manera homogénea acerca de este asunto.

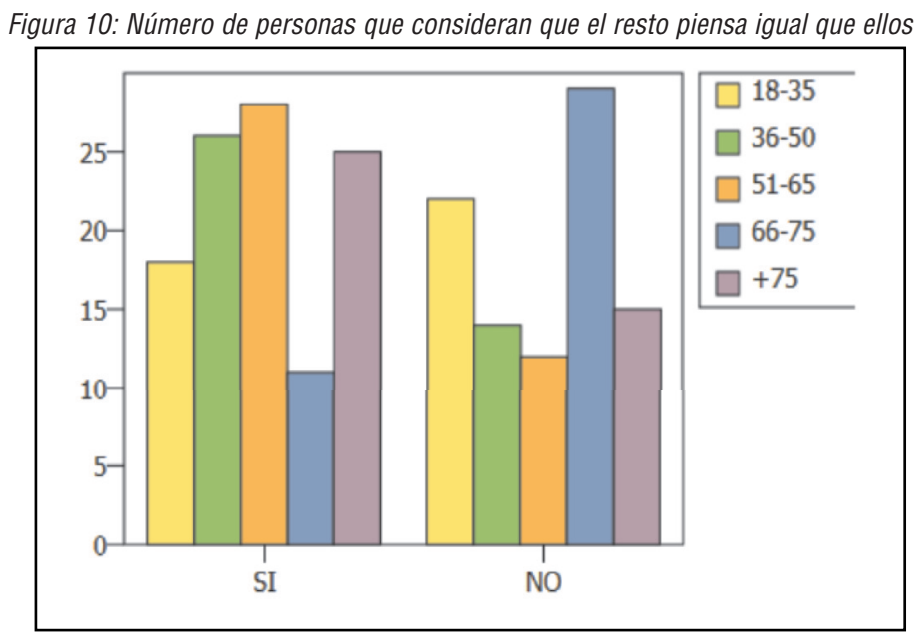

Para concluir con este análisis, se puede resumir que, en la muestra analizada, sí es cierto el hecho de que las personas más mayores piensan menos en la muerte que las más jóvenes. No obstante, un dato relevante es que son las personas incluidas en la horquilla etaria entre 51 y 65 años las que más lo hacen, más miedo tienen y más ansiedad padecen; aunque después de los 65 años este miedo y ansiedad desaparecen notablemente, puesto que alcanzar la edad de jubilación es un claro punto de inflexión en el cambio de visión sobre la muerte.

\section{CONCLUSIONES}

Con el presente trabajo, además de perseguir profundizar en el tema de la muerte y el miedo y la ansiedad que ésta puede provocar, hemos intentado encontrar la edad media a la que se produciría una aceptación de la misma, disminuyendo de esta forma los sentimientos negativos de ansiedad y miedo.

Se llega así a la conclusión de que son las personas mayores las que afrontan la muerte de una forma mucho más positiva que las personas más jóvenes.

En pleno siglo XXI, se siguen teniendo demasiados tabúes sobre acontecimientos naturales de la vida (incluida la muerte), Io cual dificulta poder hacer frente, en un futuro, a situaciones que vamos a tener que vivir de forma obligada y sin poder hacer nada al respecto. Por ello, sería conveniente comenzar a tratar estos temas como lo que son: situaciones cotidianas e irremisibles.

Una de los principales afanes del ser humano es intentar retrasar la muerte mediante su medicalización, pero no puede posponerse de forma indefinida; por ello, cuando llega es esencial que se reúnan todas las condiciones 
para que la persona sienta que puede morir en paz. Esto puede ser un factor muy importante para las personas próximas a la muerte pues, si consideran que pueden ir en paz, todo resulta más fácil a su alrededor (Gil-Juliá, Ballester-Arnal, Ruiz-Palomino y Castro-Calvo, 2017).

Con todo esto, es realmente importante resaltar un concepto que, a nuestro entender, es imprescindible en la vida de todo ser humano: hablamos de la resiliencia. En referencia a Cuellar-Castro $(2015$, p. 1), la resiliencia es "esa capacidad de afrontar y superar las vicisitudes de la vida". También es una forma de revelarse ante el sufrimiento y poder decir, de forma abierta y a los cuatro vientos, que el dolor no va a poder contigo, que todo pasa, y todo te hace (de alguna manera) más fuerte. Supone y representa una cadena de historias dolorosas que ya han sido superadas y que, mientras las estabas superando, tienes que aprender a vivir con ese dolor y hacerlo tuyo para que, en algún momento, se puedas Ilegar a convivir con él hasta superarlo. Así es que, después de eso, solo te queda una cosa, la resiliencia: Resistir, Empatizar, Sobrevivir, Seguir tu instinto, Luchar, Intentar levantarse de nuevo, nunca perder la Esperanza, ser Natural ante la vida, ponerle mucho Corazón en cada paso que des, ser Intenso en cada acción llevada a cabo y, sobre todo, y muy importante, Autoconocerte: saber cómo eres, conocerte, preguntarte qué quieres, quererte y cuidarte.

Con esto, queremos decir que cada persona es diferente, pero el afrontamiento y la superación de las situaciones difíciles se hace necesario para todas. Vivir, es lo más importante y, nos atrevemos a decir, que es lo único que tenemos que hacer en la vida. Por lo tanto, tenemos que superar con resiliencia todas las situaciones adversas que encontremos en el camino, para cumplir el papel principal que tenemos en el mundo: disfrutar viviendo.

\section{REFERENCIAS BIBLIOGRÁFICAS}

Cuellar-Castro, M. (2015). Reflexiones sobre la resiliencia. Boletín Científico. Sapiens Research GROUP, 5(1), 12.

Gil-Juliá, B., Ballester-Arnal, R., Ruiz-Palomino, E. y Castro-Calvo, J. (2017). Percepción en personas mayores de qué podría ayudarnos a morir en paz. INFAD Revista de Psicología, 1(2), 81-92.

Martínez Pascual, B., Alonso Valera, J.M. y Calvo Francés, F. (2001). Estudio comparativo de ansiedad ante la muerte en una muestra de ancianos y de jóvenes. Psiquis, 22(5), 176-182.

Real Academia Española. (2014). Diccionario de la Lengua Española (23a ed.). Consultado en https://dle.rae.es/?id=Q0MaZUb

Serra Desfilis, E. y Abengózar Torres, M.C. (1990). Ancianidad y preparación para la muerte. Anales de Psicología, 6(2), 147-158. 